\title{
Pênfigo vulgar em condição avançada na atenção básica: relato de caso
}

\author{
Pemphigus vulgaris in advanced condition in primary care: case report \\ Pénfigo vulgar en estado avanzado en atención primaria: relato de caso
}

Recebido: 22/01/2021 | Revisado: 24/01/2021 | Aceito: 25/01/2021 | Publicado: 01/02/2021

Ramon Rodrigues de Lima

ORCID: https://orcid.org/0000-0002-4493-1670

State University of Paraíba, Brasil

E-mail: ramon.va@hotmail.com

Lílian Gabriely de Sá Barreto Leite Torres ORCID: https://orcid.org/0000-0002-5157-7331

State University of Paraíba, Brasil lilian1a2013@gmail.com

Brenno Anderson Santiago Dias

ORCID: https://orcid.org/0000-0002-1047-3210

State University of Paraíba, Brasil

E-mail: brennosantiagod@gmail.com

José Martí Luna Palhano

ORCID: https://orcid.org/0000-0002-1936-0589

State University of Paraíba, Brasil

E-mail: rosemartilunapalhano1@gmail.com

Maria Izabela Brandão Vasconcelos

ORCID: https://orcid.org/0000-0002-2473-2789

State University of Paraíba, Brasil

E-mail: izabelabrandao18@gmail.com

Fábio Gabriel de Sousa Carvalho

ORCID: https://orcid.org/0000-0002-7777-4827

State University of Paraíba, Brasil

E-mail: fabio.carvalho@aluno.uepb.edu.br

Matheus Andrade Rodrigues

ORCID: https://orcid.org/0000-0003-2501-6546

State University of Paraíba, Brasil

E-mail: matheusandrade1606@gmail.com

Matheus Harllen Gonçalves Veríssimo

ORCID: https://orcid.org/0000-0003-2845-4832

State University of Paraíba, Brasil

E-mail: matheusharllen@gmail.com

Diego Tomaz Lacerda

ORCID: https://orcid.org/0000-0001-8896-061X

Universyty Maurício of Nassau, Brasil

E-mail: Dt1069016@gmail.com

Rayane Cinthia Dino do Nascimento

ORCID: https://orcid.org/0000-0001-5953-4056

Federal University of Rio Grande do Norte, Brasil

E-mail: rayanecinthia927@gmail.com

\section{Resumo}

O Pênfigo Vulgar é uma doença autoimune crônica caracterizada pela formação de bolhas na pele e mucosas, que resulta a perda de adesão entre os queratinócitos, ocasionando a desintegração das células, fenômeno denominado acantólise. Além de anticorpos imunoglobulina $\mathrm{G}(\mathrm{IgG})$ direcionados contra proteínas desmossomais. Dentre as patologias dermatológicas imunologicamente mediadas o pênfigo vulgar é a doença mais comum entre os tipos de pênfigo, tornando-se agressiva quando não diagnosticada previamente, apresentando inicialmente suas manifestações na cavidade bucal, dessa forma o Cirurgião-Dentista desempenha papel crucial no diagnóstico e manejo clínico das manifestações orais. $\mathrm{O}$ caso relatado neste artigo configura-se como um quadro de pênfigo vulgar que foi tratado e 
acompanhado, paralelamente ao tratamento médico, na unidade básica de saúde do município de Belém-PB. Contudo conclui-se que é importe o cirurgião dentista tenha conhecimento para o reconhecimento das doenças autoimunes, especificamente o pênfigo vulgar, visto que o diagnóstico precoce é essencial para o tratamento da patologia, evitando transtornos sistêmicos posteriores. Além de enfatizar o papel da abordagem odontológica e a importância da atuação multiprofissional.

Palavras-chave: Pênfigo vulgar; Unidade básica de saúde; Manifestações orais.

\begin{abstract}
Pemphigus Vulgaris is a chronic autoimmune disease characterized by the formation of blisters on the skin and mucous membranes, which results in the loss of adhesion between keratinocytes, causing the disintegration of cells, a phenomenon called acantholysis. In addition to immunoglobulin G (IgG) antibodies directed against desmosomal proteins. Among immunological mediated dermatological pathologies, pemphigus vulgaris is the most common disease among pemphigus types, becoming aggressive when not previously diagnosed, initially presenting its manifestations in the oral cavity, thus the Dental Surgeon plays a crucial role in the diagnosis and management clinical presentation of oral manifestations. The case reported in this article is configured as a condition of pemphigus vulgaris that was treated and followed up, in parallel with medical treatment, at the basic health unit in the municipality of Belém-PB. However, it is concluded that it is important for the dentist to have knowledge for the recognition of autoimmune diseases, specifically pemphigus vulgaris, since early diagnosis is essential for the treatment of the pathology, avoiding posterior systemic disorders. In addition to emphasizing the role of the dental approach and the importance of multiprofessional performance.
\end{abstract}

Keywords: Pemphigus vulgaris; Basic health unit; Oral manifestations.

\title{
Resumen
}

El pénfigo vulgar es una enfermedad autoinmune crónica caracterizada por la formación de ampollas en la piel y membranas mucosas, que resulta en la pérdida de adhesión entre los queratinocitos, provocando la desintegración de las células, fenómeno llamado acantólisis. Además de anticuerpos de inmunoglobulina G (IgG) dirigidos contra proteínas desmosomales. Entre las patologías dermatológicas inmunológicas mediadas, el pénfigo vulgar es la enfermedad más común entre los tipos de pénfigo, volviéndose agresivo cuando no se diagnostica previamente, presentando inicialmente sus manifestaciones en la cavidad bucal, por lo que el Cirujano Dentista juega un papel crucial en el diagnóstico y manejo. presentación clínica de manifestaciones orales. El caso denunciado en este artículo se configura como una condición de pénfigo vulgar que fue atendido y seguido, en paralelo con el tratamiento médico, en la unidad básica de salud del municipio de Belém-PB. Sin embargo, se concluye que es importante que el odontólogo tenga conocimientos para el reconocimiento de enfermedades autoinmunes, específicamente el pénfigo vulgar, ya que el diagnóstico precoz es fundamental para el tratamiento de la patología, evitando trastornos sistémicos posteriores. Además de enfatizar el papel del abordaje odontológico y la importancia del desempeño multiprofesional.

Palabras clave: Pénfigo vulgar; Unidad básica de salud; Manifestaciones bucales.

\section{Introdução}

O pênfigo consiste em um grupo de doenças raras e autoimune caracterizada por bolhas epiteliais que afeta a pele e/ou as mucosas (Mota et al., 2020). Entre os vários subtipos amplamente descritos de pênfigo, o pênfigo vulgar (PV) e pênfigo foliáceo (PF) são as duas formas mais comuns. (Siu, Landon \& Ramos, 2015; Veja-Menréje et al., 2015; Buonavoglia et al., 2019).

Embora nenhuma etiologia clara tenha sido estabelecida para essas doenças, a complexa patogênese da PV envolve a geração de autoanticorpos contra proteínas conjuntivas da pele e mucosa, incluindo caderinas desmossomais (Tavakolpour, 2017; Mays, et al., 2020). A desmogleína 3 é o principal antígeno, mas 50-60\% dos pacientes também têm anticorpos antidesmogleína 1 (Cozzani et al., 2013; Kasperkiewics et al., 2017; Mays et al., 2020). O claro papel dos autoanticorpos na patogênese da PV sugere um importante papel terapêutico para agentes direcionados que bloqueiam a geração ou sobrevivência de componentes imunológicos autorreativos (Ellebrecht \& Payne, 2017; Buonavoglia et al., 2019; Mays et al., 2020). 
As respostas imunes agressivas e aberrantes contribuem para o desenvolvimento e progressão do pênfigo por meio de diferentes mecanismos, incluindo a produção de autoanticorpos pelas células B do plasma, a atividade de células T auxiliares (Th) CD4 + autorreativas, e Células T CD8 +. Este grupo raro de doenças pode ser induzido ou exacerbado por fatores genéticos e exógenos (Tavakolpour, 2017; Deyhimi e Alishahi, 2018; Buonavoglia et al., 2019).

Embora epidemiologicamente não haja evidência de predileção por gênero, alguns estudos relataram uma ligeira prevalência em mulheres. Todas as idades podem ser afetadas, embora o maior número de casos seja observado em pacientes na faixa dos 40 e 50 anos (Saccucci et al., 2018).

A maioria dos agentes causadores da estimulação do sistema imunológico pode ser considerada uma ameaça potente para pacientes com pênfigo ou mesmo indivíduos suscetíveis. As lesões parecem ser desencadeadas por diferentes fatores são eles: alterações hormonais, drogas, doenças, dieta, gravidez, estresse emocional ou vários outros fatores ambientais (Tavakolpour, 2017; Saccucci et al., 2018).

Os primeiros sinais da doença, em mais de 50\% dos casos, surgem na mucosa oral (Nascimento et al., 2020). Embora não haja predileção por área, as lesões podem estar localizadas na mucosa bucal, palato mole, lábio inferior, língua e, com menos frequência, na gengiva (Saccucci et al., 2018; Mays, et al., 2020). Essas lesões orais podem variar de úlceras bastante superficiais, a pequenas vesículas ou bolhas. Na cavidade oral, as bolhas rompem-se rapidamente, deixando uma erosão dolorosa com sensação de queimação. O tamanho das úlceras é extremamente variável (Saccucci et al., 2018). Quando as bolhas se rompem, as úlceras resultantes são frequentemente grandes e podem coalescer. As lesões podem variar do tamanho de uma pequena úlcera aftosa $(<5 \mathrm{~mm})$ a uma lesão grande, dolorosa, coberta por psuedomembrana $(2 \mathrm{~cm})$. A gravidade das lesões orais pode impedir uma nutrição adequada devido à dor intensa durante o consumo de alimentos (Siu, Landon \& Ramos, 2015).

O diagnóstico requer uma biópsia para identificar a localização da separação epitelial (acantólise) e, geralmente, imunofluorescência direta para localizar o autoanticorpo ligado ao o tecido nas áreas suprabasais do estrato espinhoso (IgG e C3). O elemento semiotécnico para um diagnóstico mais simples do pênfigo vulgar é a observação do resultado positivo do sinal de Nikolsky, manobra essa que consiste no desprendimento da mucosa epitelial a partir de uma ligeira fricção, expondo o tecido conjuntivo e dando origem a uma úlcera (Saccucci et al., 2018; Mays et al., 2020; Siu, Landon e Ramos, 2015).

Em suma, o presente artigo tem como objetivo relatar um caso clínico de diagnóstico de pênfigo vulgar feito na unidade básica de saúde no município de Belém no estado da Paraíba.

\section{Metodologia}

O presente trabalho trata-se de um relato de caso e análise de literaturas já publicadas, em certas bases de dados eletrônicas: SciELO (Scientific Electronic Library Online), PUBMED (U. S. National Library of Medicine (NLM), LILACS (Literatura Latino-americana e do Caribe em Ciências da Saúde) e BVS (Biblioteca Virtual de Saúde). A fim de coletar o máximo de informações possíveis para que fosse desenvolvido o relato com maior fidelidade nas afirmações.

Os critérios de inclusão para a produção deste estudo constituem 14 trabalhos científicos, tanto produções nacionais quanto internacionais, em inglês e português, divulgados entre os anos 2015 a 2020. Assim, foram coletados artigos que abordam o pênfigo vulgar associado a prática clínica odontológica em Unidades Básicas de saúde (UBS) para posterior comparação com o caso clínico apresentado.

Para coleta dos artigos, os descritores utilizados foram "Pênfigo vulgar", "Unidade básica de saúde”, "Manifestações orais". A pesquisa foi realizada através de meios eletrônicos, devido a facilidade de acesso, empregando os critérios de inclusão supracitados, obtendo os artigos de forma precisa, onde, nas bases de dados selecionadas, foram adaptadas tais palavras-chaves como forma de evitar pequenos vieses durante o referido estudo. 
Trata-se de um estudo descritivo, retrospectivo, qualitativo, feito por meio da técnica de observação direta (Pereira et al., 2018), sendo o pesquisador o instrumento primordial. Seguindo os princípios éticos, o paciente consentiu com a divulgação dos dados e exibição de imagens de seu caso para finalidades científicas por meio da assinatura de um Termo de Consentimento Livre e Esclarecido.

\section{Relato de Caso}

Paciente do sexo masculino, melanoderma, 37 anos de idade, diabético e portador de hipertensão arterial, fazendo uso de insulina humana nph 100ui/ml, insulina humana regular 100ui/ml, para controle da diabete, captopril de $25 \mathrm{mg}$, hidroclorotiazida de $25 \mathrm{mg}$, besilato de anlodipino de $10 \mathrm{mg}$. para controle da hipertensão arterial e dieta alimentar. Compareceu à Unidade Básica de Saúde (UBS) do município de Belém-PB, relatando dificuldade de deglutição, febre, mal estar, lesões erosivas dolorosas espalhadas por toda cavidade bucal e faringe, por um período de 15 dias. O paciente relatou o surgimento primário de bolhas que facilmente eram rompidas expondo uma região dolorosa. Além disso, também foram relatadas, a presença de lesões corporais nas axilas, nas costas, braços e região genital, com sintomatologia. Durante a ectoscopia, o exame extrabucal identificou gânglios cervicais infartados, sintomáticos e móveis.

O exame clinico intraoral revelou a presença de múltiplas lesões e úlceras extensas, doloridas, superficiais, irregulares, eritematosas, com exsudato sanguinolento (Figuras 1, 2, 3, 4 e 5), dificuldade de fonação, deglutição, mastigação e sinal de Nikolsky positivo (Figura 1). Um exame detalhado revelou halitose intensa.

Figura 1 - Múltiplas lesões extensas em exame extrabucal, impossibilitando a abertura bucal.

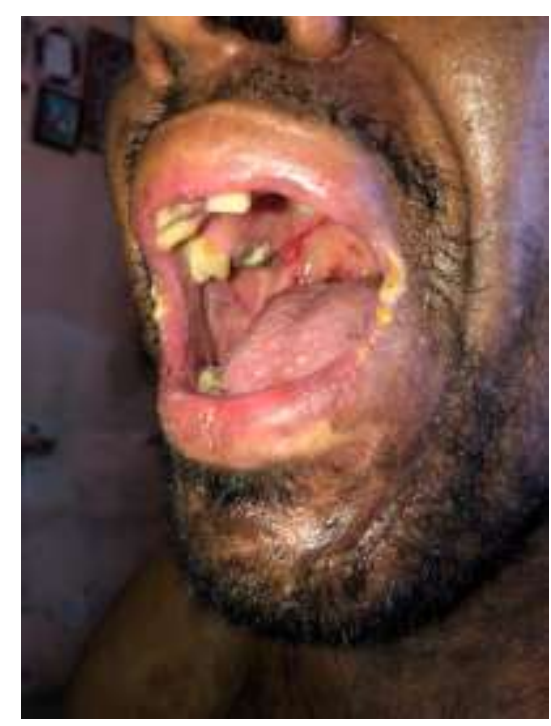

Fonte: Autores (2021) 
Figura 2 - Lesões no ângulo da boca as quais dificultava a abertura bucal, causando dificuldade para alimentação.

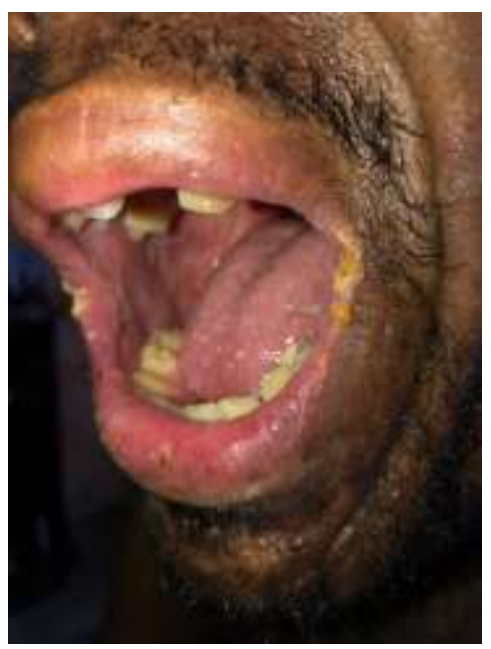

Fonte: Autores (2021)

Figura 3 - Presença de lesões no ângulo da boca e na mucosa jugal.

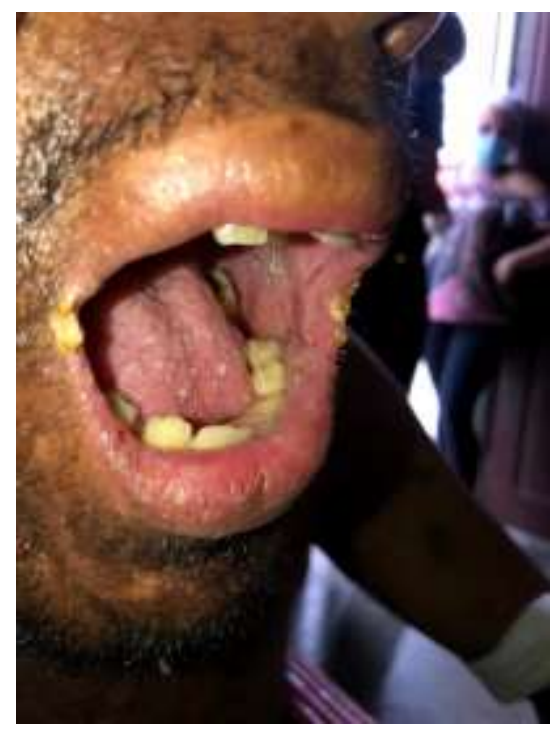

Fonte: Autores (2021)

Por estar com a cavidade oral muito acometida o paciente não conseguia uma abertura de boca para realização de biopsia intraoral. Realizaram-se, então, junto a equipe da UBS, uma biópsia excisional na região inguinal para exame histopatológico. O procedimento foi realizado em várias regiões do corpo devido à sensibilidade dos tecidos e à complexidade na remoção de um fragmento de pele que contivesse uma espessura adequada. O diagnóstico histopatológico final foi de pênfigo vulgar, porém sem a presença de sinais de malignidade.

Diante do quadro apresentado, onde se tinha lesões agressivas e dolorosas a cometendo quase todo o corpo (Figura 4 e 5) e por apresentar um quadro agudo das lesões o paciente foi encaminhado para o Hospital Universitário Lauro Wanderley na cidade de João Pessoa-PB. 
Figura 4 - Lesões em todo o corpo (vista dorsal), após um mês de tratamento.

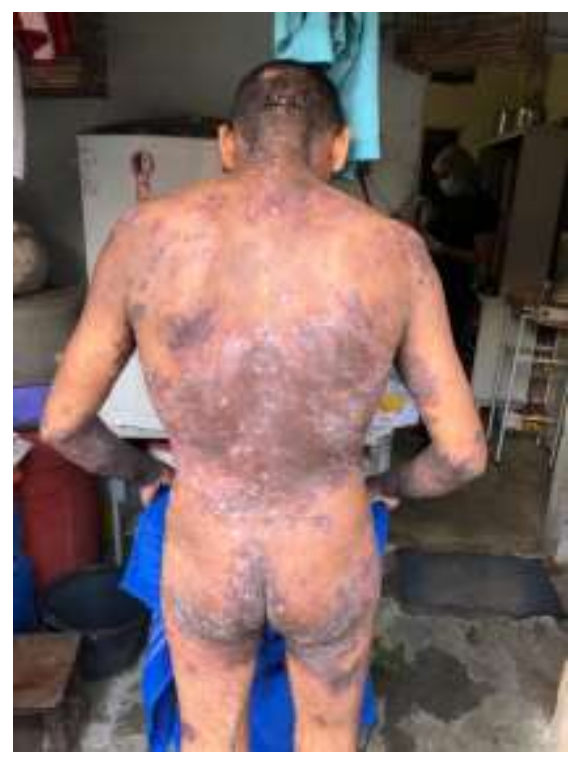

Fonte: Autores (2021)

Figura 5 - Lesões em todo o corpo (vista ventral), situação encontrada quando o paciente procurou a unidade básica de saúde.

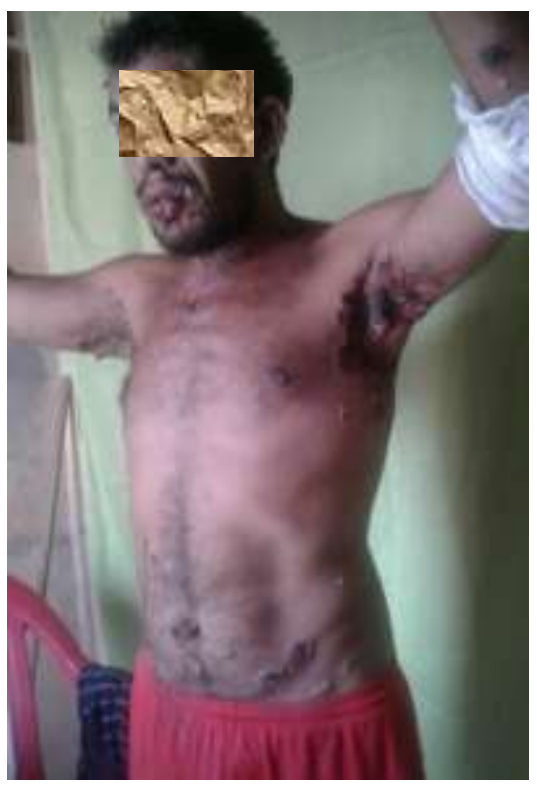

Fonte: Autores (2021)

No Hospital Universitário Lauro Wanderley foi medicado sistemicamente com prednisona 20mg, azatiopina 50mg e hixizine $25 \mathrm{mg}$ e também com a medicação para controle da diabetes e hipertensão arterial. Para as lesões foi prescrito clobetazol creme para aplicar nas lesões bolhosas e diprogenta para as lesões orais.

Resolvido o episódio agudo das manifestações orais e ter recebido alta do hospital o paciente voltou para sua residência na cidade de Belém-PB o paciente permaneceu em tratamento do pênfigo vulgar bem como do diabetes e hipertensão arterial, o qual passou a ser acompanhado pela equipe de saúde da família. Quatro meses depois o paciente se apresenta com um quadro estável e sem lesão. 


\section{Discussão}

O pênfigo é uma patologia autoimune caracterizada pelo surgimento de bolhas, ulcerações na pele e membranas mucosas. Existem algumas variantes do pênfigo, entretanto, o pênfigo vulgar é a manifestação mais comum. Shamim et al. (2008), apontam que essa variante do pênfigo ocorre com prevalência entre a $5^{\mathrm{a}}$ e $6^{\mathrm{a}}$ décadas de vida, sendo que alguns casos raros já foram registrados em crianças e idosos. Ademais, salientam que a incidência do pênfigo vulgar sobre a população é considerada rara, pois cerca de 0,5 a 3,2 casos são notificados a cada 100.000 habitantes no período de um ano. Segundo Bascones-Martínez et al. (2015), mesmo não havendo predileção por gênero, ocorre uma ligeira prevalência da doença em mulheres.

O paciente acompanhado no estudo apresentou uma doença de curso rápido e agressivo com lesões na cavidade bucal e, posteriormente, apareceram lesões cutâneas. Na literatura de Ibsen \& Phelan, (2009), as lesões cutâneas surgem após as manifestações orais, em consonância com as pesquisas de Dagistan et al. (2008), que afirma que de 70 a $90 \%$ dos casos de pênfigo a sua manifestação primária ocorre na mucosa da boca do paciente. Sendo assim, as lesões podem estar localizadas em qualquer parte da cavidade oral, sendo mais comumente encontradas em áreas sujeitas a traumas de fricção, como a mucosa da bochecha, faringe, laringe, esôfago, mucosa genital e sem descartar a possibilidade de surgirem erupções cutâneas simples para erosões, vesículas, bolhas ou úlceras na própria pele do paciente.

No relato em questão, o paciente observado apresentou Sinal de Nikolsky positivo, uma manifestação típica do pênfigo vulgar, que consiste na aplicação de pressão na pele próxima a uma lesão, ocasionando o surgimento de uma nova bolha devido à acantólise (Nascimento et al. 2020). Contudo, segundo estudos de Uzun \& Durdu (2006), resultados falsos negativos são possíveis, desse modo, foi realizado um exame complementar de biópsia excisional no paciente acompanhado para confirmação da patologia.

No caso relatado, a opção de tratamento foram os corticosteroides sistêmicos e tópicos, prednisona e clobetasol, assim como o imunossupressor azatioprina. Virgolini \& Marzocchi, (2003), sugerem a combinação desses corticosteroides e imunossupressores no tratamento de pênfigo vulgar. Além disso, estudos realizados por Mays et al. (2020), afirmam que tratamentos alternativos estão sendo utilizados com agentes biológicos como o rituximabe em pacientes com pênfigo vulgar moderado a grave, entretanto, apesar dos avanços, pesquisas devem ser realizadas para comprovação da terapia biológica. Somado a isso, Yousefi et al. (2017) recomendam a terapia a laser de baixa intensidade (LBI) no tratamento de pacientes de pênfigo vulgar com lesões recalcitrantes em que os recursos terapêuticos convencionais não são suficientes.

O acompanhamento do caso evidenciou a importância de uma equipe multidisciplinar no tratamento de pacientes com pênfigo vulgar, assim como, foi realizado nos estudos de Yousefi et al. (2017), em que o diagnóstico final foi obtido pela multidisciplinaridade profissional por meio de um médico dermatologista que promoveu o exame histopatológico e a avaliação de imunofluorescência direta.

Após quatro meses o paciente supervisionado apresentou melhoras em seu quadro clínico e sem ulcerações, assim como no caso relatado por Arpita et al. (2015), após o uso da prednisona associada ao enxaguatório analgésico tópico e pomada de acetonido de triancinolona $0,1 \%$, em que o paciente apresentou melhora gradual das ulcerações com $90 \%$ das lesões curadas a partir do terceiro atendimento.

\section{Considerações Finais}

O pênfigo vulgar é uma doença vesicobolhosa, rara, grave e de natureza autoimune, ocasionada pelo direcionamento de autoanticorpos contra proteínas de adesão celular da pele e membranas mucosas em que, muitas vezes, os sintomas primários surgem na cavidade bucal e, posteriormente, podem se espalhar para o restante do corpo. 
Somado a isso, o diagnóstico exige atuação multidisciplinar dos profissionais de saúde como Cirurgiões-Dentistas e médicos dermatologistas, nos quais, os primeiros têm papel fundamental na identificação precoce da enfermidade o que possibilita o aumento de maiores chances de remissão completa da doença. Por causa disso, fica evidente a importância da qualificação do odontólogo no diagnóstico das manifestações clínicas orais de pênfigo vulgar.

Logo, este relato de caso, abordou uma ocorrência complexa e avançada dessa patologia em que o paciente foi encontrado em estado precário e apresentou lesões bolhosas na cavidade oral e na pele. Diante disso, diversas medidas terapêuticas foram utilizadas para estabilizar o quadro clínico do enfermo, resultando assim no alívio e remissão da doença no período de quatro meses.

\section{Referências}

Arpita R. et al. (2015). Oral Pemphigus Vulgaris: Case Report. Ethiopian-Journal of Health Sciences, 25 (4): $367-372$.

Bascones-Martínez, A. et al. (2015). Immune-mediated diseases: what can be found in the oral cavity? International Journal of Dermatology, 54(3): 258-270.

Buonavoglia, A. et al. (2019). Pemphigus and mucous membrane pemphigoid: An update from diagnosis to therapy. Autoimmunity Reviews, 18 (4): 349-358.

Cozzani E. et al. (2013). Are clinical phenotype and autoantibody profile always concordant in pemphigus? A study in a cohort of pemphigus patients. European Journal of Dermatology, 23(1), 40-48.

Dagistan, S. et al. (2008). Oral Pemphigus Vulgaris: A Case Report with review of literature. Journal of Oral Science, 50(3):359-62.

Deyhimi, P. \& Alishahi, B. (2018). Study of Extrinsic Apoptotic Pathway in Oral Pemphigus Vulgaris Using TNFR 1 and FasL Immunohistochemical Markers and TUNEL Technique. Journal of Shiraz University Medical Sciences, 19(2): 132-141.

Ibsen, O. A. C. \& Phelan, J. A. (2009) Oral Pathology for Dental Hygienist. Saunders (W.B.) Co Ltd [ebook], 5th edition.

llebrecht C.T. \& Payne A.S. (2017). Setting the target for pemphigus vulgaris therapy. JCI Insight, 2(5): e92021.

Mays, J. W. et al. (2020). WWOM VII: A systematic review of immunobiologic therapy for oral manifestations of pemphigoid and pemphigus. Oral Disease, 25(1): 111-121.

Mota, M. S. et al. (2020). Theoretical-practical reflection of multiprofessional assistance to a patient with pegphic vegetant. Research, Society and Development, 9(7): e-32973786.

Nascimento, M. A. et al. (2020). Cuidado multiprofissional ao paciente portador de pênfigo vulgar. Research, Society and Development, 9(8), e-778986596.

Saccucci, M. et al. (2018). Autoimmune Diseases and Their Manifestations on Oral Cavity: Diagnosis and Clinical Management. Journal of Immunology Research, 2018: 6061825.

Shamin, T. et al. (2008). Pênfigo vulgar em cavidade oral: análise clínica de 71 casos. Medicina Oral Patologia Oral y Cirugia Bucal, 13 (10): E622-6.

Siu, A., Landon, K. \& Ramos, D. M. (2015). Differential diagnosis and management of oral ulcers. Seminars in Cutaneous Medicine and Surgery, 34: 171177.

Tavakolpour, S. (2018). Pemphigus trigger factors: special focus on pemphigus vulgaris and pemphigus foliaceus. Springer-Verlag GmbH Germany. Archives of Dermatological Research, 310 (2): 95-106.

Vega-Memíje1, M. E. et al. (2015). Is there a causal relationship between HSV-1 and pemphigus vulgaris? SpringerPlus, 4: 811.

Virgolini, L. \& Marzocchi, V. (2003). Anti-CD20 monoclonal antibody (rituximab) in the treatment of autoimmune diseases. Successful result in refractory Pemphigus vulgaris: report of a case. Haematologica, 88(7): ELT24.

Uzun S. \& Durdu, M. (2006). The specificity and sensitivity of Nikolskiy sign in the diagnosis of pemphigus. Journal of the American Academy of Dermatology, 54(3):411-5.

Yousefi, M. et al. (2017). The Effect of Low Level Laser Therapy on Pemphigus Vulgaris Lesions: A Pilot Study. Journal of Lasers in Medical Sciences, 8(4): $177-180$. 